Dossier: V Simposio Internacional y IX Nacional sobre Derechos de la Niñez y la Adolescencia

\title{
La infancia generalizada. Tendencias y retos actuales en la evaluación de las dificultades del desarrollo infantil
}

\author{
Milagros Jaime Arteaga \\ Universidad de Costa Rica, Costa Rica \\ milagros.jaime@gmail.com \\ https://orcid.org/0000-0003-3092-3642
}

Recibido: 1 de julio de 2020

Aceptado: 31 de setiembre de 2020

Resumen: Este trabajo propone una reflexión sobre los contextos contemporáneos y su influencia en los problemas más frecuentes de la infancia en la actualidad. Se analizan las brechas existentes entre norma jurídica y realidad social en Costa Rica, retomando el concepto de "Biopolítica de la infancia" (Bustelo, 2005). Desde el marco del enfoque de derechos y la definición de violencia contra los niños, niñas y adolescentes de la OMS, se propone la idea de una infancia generalizada en nuestro tiempo para describir la tendencia a homologar científicamente los padecimientos infantiles. Siguiendo la noción de evaluación generalizada expuesta por Najles (2010), se describen los riesgos de patologizar y homogenizar a la hora de realizar un abordaje actual de las dificultades de la infancia. Ante estos retos, se plantea la necesidad de mantener una dimensión de desconocimiento en el saber sobre la infancia que permita incluir la singularidad de cada niño y cada niña.

Palabras clave: infancia; derechos; dificultades de la infancia; evaluación infantil; patologización.

\section{Generalized Childhood, Current Trends and Challenges in the Evaluation of Childhood Development}

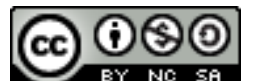

La Revista Estudios es editada por la Universidad de Costa Rica y se distribuye bajo una Licencia Creative Commons Atribución-NoComercial-CompartirIgual 3.0 Costa Rica. Para más información envíe un mensaje a 


\title{
Dossier: V Simposio Internacional y IX Nacional sobre Derechos de la Niñez y la Adolescencia
}

\begin{abstract}
This paper proposes a reflection on contemporary contexts and their influence on the most frequent childhood difficulties of today. It examines existing gaps between the law and social reality in Costa Rica, going back to the concept of "biopolitics of childhood" (Bustelo, 2005). From a rights-based approach and using the WHO's definition of violence against children and adolescents, it proposes the idea of a generalized childhood in our times to describe the trend to standardize childhood difficulties scientifically. Following the notion of generalized evaluation expressed by Najles (2010), it describes the risks of pathologization and standardization in the way childhood difficulties are addressed today and calls for a need to maintain a dimension of ignorance in the knowledge of childhood that allows for accommodating each child's uniqueness.
\end{abstract}

Keywords: childhood; rights; childhood difficulties; childhood evaluation; pathologization

\section{Introducción}

Se sabe que la infancia no es una entidad dada por sí misma, sino que se construye en cada momento histórico, en cada lugar y contexto. Su existencia es simbólica: se encuentra en el discurso social, en las diversas miradas y representaciones que como sociedad tenemos sobre los niños y las niñas. Cada generación no inicia su recorrido por la vida desde cero. Por el contrario, los infantes adquieren una historia previa y deben apropiarse de las significaciones que les preceden, de los lugares que les son ofrecidos y de las modalidades de lazo social propias de su época, para construir sus posibilidades singulares de ser en el mundo al cual pertenecen. Sería más exacto hablar de las infancias, a fin de dar cuenta de las diversas realidades que constituyen el ser infantil.

Por lo tanto, pensar las infancias actuales y sus dificultades nos obliga a la reflexión sobre las coordenadas de nuestra contemporaneidad y de las condiciones de vida de los niños y las niñas, con el fin de situar la infancia más que enfocarla de una manera general. El presente escrito propone un acercamiento a los contextos actuales en los que se sitúan las infancias en América Latina y Costa Rica, para

\section{(c) (1) (2)}

La Revista Estudios es editada por la Universidad de Costa Rica y se distribuye bajo una Licencia Creative Commons Atribución-NoComercial-CompartirIgual 3.0 Costa Rica. Para más información envíe un mensaje a 
Dossier: V Simposio Internacional y IX Nacional sobre Derechos de la Niñez y la Adolescencia proponer algunas discusiones sobre las principales dificultades del desarrollo infantil, así como los retos en su evaluación y abordaje.

\section{Contextos contemporáneos del devenir infantil}

La primera coordenada de nuestra contemporaneidad que propone analizar, la más evidente y generalizada, es el acelerado avance tecnológico sin precedentes que se introduce en todas las esferas de la vida. La UNICEF dedicó el Estado Mundial de la Infancia del año 2017 al análisis de la infancia en el mundo digital, destacando la manera en que la tecnología ha transformado el mundo en el que vivimos, y en particular, cómo está cambiando cada vez más a la infancia (UNICEF, 2017). Este documento resalta que:

La rápida proliferación de la tecnología de la información y las comunicaciones (TIC) es una fuerza imparable que afecta prácticamente a todas las esferas de la vida moderna, desde las economías a las sociedades y las culturas... y a la vida cotidiana (UNICEF, 2017 p. 8).

Como nunca en la historia, hoy en día las personas hacen uso de artefactos y espacios que tienen la característica de ser personales y privados. Esto responde a una lógica de consumo y de individualismo que vuelve a las personas cada vez más ensimismadas y extrañas a lo ajeno, algo así como una manera de vivir al margen de la sociedad. La paradoja es que las Tecnologías de la Información y la Comunicación, de ahora en adelante TIC, que proponen vivir conectado a las "redes sociales" en realidad aísla más de lo que une. Este fenómeno va de la mano con otras características de esta época, el valor del éxito y la destrucción del capital simbólico que se produce por la lógica capitalista.

El éxito, entendido desde un modelo de competencia e individualismo alrededor del consumo, es un valor que ejerce un influjo determinante en los vínculos sociales contemporáneos. Cabe preguntarse hasta qué punto este modelo

\section{(c) (i) (2)}

La Revista Estudios es editada por la Universidad de Costa Rica y se distribuye bajo una Licencia Creative Commons Atribución-NoComercial-CompartirIgual 3.0 Costa Rica. Para más información envíe un mensaje a 


\section{Dossier: V Simposio Internacional y IX Nacional sobre Derechos de la Niñez y la Adolescencia}

se filtra en las preocupaciones que como sociedad nos planteamos respecto a lo que los niños y las niñas necesitan alcanzar para tener mejores oportunidades en su vida futura, o sobre los criterios que se utilizan para valorar el éxito de un niño o una niña en su adaptación al entorno y las exigencias que de ellos se derivan.

Por otro lado, el orden capitalista contemporáneo, con sus reglas y patrones de consumo en el contexto neoliberal, propone una lógica en las relaciones humanas que se opone al fortalecimiento del capital simbólico. La psicoanalista Colette Soler (2010), quien, tomando el concepto de capital simbólico del sociólogo francés Pierre Bourdieu, explica:

El capital simbólico comprende el conjunto de los saberes que se transmiten, y que constituyen armas para el futuro, son instrumentos posibles del éxito; pero el capital simbólico incluye otra cosa: los valores estéticos, morales, religiosos; los valores que dan sentido a las desgracias de la vida, que las compensan, que permiten soportarlas; y el discurso capitalista destruye estos valores, porque conoce solo un valor: la mercancía. (Soler, 2010, p. 20-21).

De esta manera, al debilitarse el capital simbólico, se pierde la posibilidad de que funcione como soporte para construir una identidad social y defender un valor personal que no sea solo el éxito individualista. El filósofo surcoreano Byung-Chul Han, uno de los más fuertes críticos contemporáneos de la sociedad del hiperconsumo, propone un enfoque muy pertinente sobre el exceso de este empuje hacia el éxito individualista de nuestra época. Han describe a la sociedad actual como sociedad del cansancio, caracterizada por la auto explotación, que produce adultos permanentemente agotados bajo el imperativo del rendimiento y el éxito personal. Para este filósofo "la sociedad del siglo XXI ya no es disciplinaria [como describe Foucault], sino una sociedad de rendimiento. Tampoco sus habitantes se llaman ya 'sujetos de obediencia', sino 'sujetos de rendimiento'... emprendedores de sí mismos" (Han, 2012, p.25).

\section{() $100(0$}

La Revista Estudios es editada por la Universidad de Costa Rica y se distribuye bajo una Licencia Creative Commons Atribución-NoComercial-CompartirIgual 3.0 Costa Rica. Para más información envíe un mensaje a 


\section{Dossier: V Simposio Internacional y IX Nacional sobre Derechos de la Niñez y la Adolescencia}

De la mano de la sociedad del rendimiento está la masificación, que hace cada vez más difícil el encuentro con la extrañeza del otro, con su particularidad y diferencia. No sorprende entonces que esta sea una época marcada por la soledad y la depresión. La Organización Mundial de la Salud, de ahora en adelante OMS, viene alertando desde hace varios años sobre el aumento de la depresión a nivel mundial. Durante la última década su incidencia se incrementó por encima del 18\%, ubicándola como la principal causa de problemas de salud y discapacidad en el mundo (Organización Mundial de la Salud/Organización Panamericana de la Salud, 2017). Sin negar las disposiciones individuales, es válido considerar las condiciones de los contextos actuales que favorecen el aislamiento y la incidencia de los estados depresivos.

Este panorama muestra contextos para el devenir infantil caracterizados por la abundancia de las TIC, con su consecuente exceso de estímulos y atención dispersa, entre adultos aislados y cansados, con poco tiempo para el diálogo y la construcción de colectividad, ¿cómo se ampara entonces a la infancia en estos tiempos?

\section{Entre la norma jurídica y la realidad social}

A 30 años de la Convención sobre los Derechos del Niño se observa que pese a los importantes avances que se promovió en las legislaciones y la creación de instituciones especializadas, estos no han demostrado ser garantía suficiente de protección para todos los niños y las niñas. El VIII Estado de los Derechos de la Niñez y la Adolescencia en Costa Rica señala que a pesar de que la Convención topó con suelo fértil, uno de los derechos más elementales de los niños, niñas y adolescentes que continúa sin cumplirse es el de vivir sin violencia (UNICEF-UCR, 2015, p. 73). Este informe expone que "existen importantes limitaciones en las competencias de crianza por parte de las personas encargadas de ella, lo que favorece el uso del castigo físico, al no contarse con un repertorio de estrategias no

\section{(c) (i) (-)}

La Revista Estudios es editada por la Universidad de Costa Rica y se distribuye bajo una Licencia Creative Commons Atribución-NoComercial-CompartirIgual 3.0 Costa Rica. Para más información envíe un mensaje a 
Dossier: V Simposio Internacional y IX Nacional sobre Derechos de la Niñez y la Adolescencia

violentas y eficaces" (UNICEF-UCR, 2015, p. 73-74). En cuanto a los niños, niñas y adolescentes en riesgo social considera una "fuerte alarma" el hecho de que muchos de ellos en situación mayor vulnerabilidad son "internados en instituciones y no en centros de acogimiento familiar" (UNICEF-UCR, 2015, p.74).

En un reportaje del periódico La Nación, la directora del Hospital Nacional de Niños reveló que, mientras en el 2006 se registraban 2,5 casos diarios de infantes valorados por manifestaciones de violencia, en el 2018 esta cifra aumentó a 5 casos por día, siendo la población más afectada los niños y niñas más pequeños, entre cero y cuatro años (Matarrita, 2019). Esta prevalencia de agresiones hacia los infantes de menor edad se refleja también en un estudio realizado por Vargas (2012) sobre muertes por agresión infantil en Costa Rica. Sus resultados revelan que entre los años 2009 y 2011 la tasa de mortalidad por homicidio en menores de 13 años se cuadriplicó, con predominio de lactantes y menores de 4 años.

Por otra parte, el VIII Estado de los Derechos de la Niñez y la Adolescencia hace una denuncia sobre las dificultades que muestran las instituciones encargadas de velar por el bienestar de la infancia, para hacer efectivos sus derechos fundamentales. Este informe afirma que en América Latina existen niños, niñas y adolescentes que enfrentan condiciones de desigualdad, pobreza, exclusión y abandono:

Todo debido a la pasividad y desinterés de los gobiernos, la deficiencia y la falta de coordinación de las instituciones encargadas, la intransigencia para hacer una realidad la perspectiva de derechos amparándose en el viejo esquema de las personas menores de edad como incapaces, y la imposibilidad para resolver problemas estructurales como la pobreza y el pleno acceso a la educación (UCR-UNICEF, 2015, p. 33).

También señala este informe que en Costa Rica uno de cada tres niños es pobre, sin dejar de alertar sobre las consecuencias de esta condición en su desarrollo futuro (UCR-UNICEF, 2015, p. 76). Pese al reconocimiento nacional e

La Revista Estudios es editada por la Universidad de Costa Rica y se distribuye bajo una Licencia Creative Commons Atribución-NoComercial-CompartirIgual 3.0 Costa Rica. Para más información envíe un mensaje a 
Dossier: V Simposio Internacional y IX Nacional sobre Derechos de la Niñez y la Adolescencia internacional y a los avances formales en temas de derechos de la infancia, aún existen importantes brechas entre norma jurídica y realidad social. Estas fallas representan también formas de violencia contra la infancia, violencias más sutiles y desapercibidas, aunque no por ello menos perniciosas.

Se observan múltiples realidades que enfrentan los niños, niñas y adolescentes en este país en donde se hace evidente este desfase en cuanto a la aplicación de la legislación vigente. Ejemplos de ello es la exclusión que siguen viviendo las personas con discapacidad pese a la existencia de la Ley 7600, las deficiencias en el debido cumplimiento de la Ley 9220 que crea la Red Nacional de Cuido y Desarrollo Infantil (UNICEF-UCR, 2015, p.75) y el lento crecimiento y especialización de la institucionalidad pública en comparación con la amplia serie de leyes aprobadas en los últimos 25 años (UNICEF-UCR, 2015, p.80).

El politólogo Eduardo Bustelo, tenaz defensor de la protección de los niños y las niñas, con amplia experiencia en el campo de las políticas sociales sobre infancia, ofrece una definición para analizar esta distancia entre norma jurídica y realidad social, en lo que él denominó la biopolítica de la infancia (Bustelo, 2005). Para describirla, sigue a dos importantes teóricos sociales: Michael Foucault y Giorgio Agamben. De Foucault toma el concepto de biopolítica (el cual describe el anudamiento entre política y vida, como una forma en que el poder se entreteje con dispositivos que inciden sobre el control del cuerpo, la construcción de la subjetividad y las formas de vida), para aplicarlo al campo de la infancia, ya que considera que el inicio de la vida es donde el biopoder "aparece en su forma paroxística" (Bustelo, 2005, p. 255).

Con Agamben, Bustelo va a señalar la diferencia entre la infancia circunscrita a la pura sobrevivencia (zoé) y las posibilidades reales de los niños y las niñas de ejercer la ciudadanía y la política (bios) (Bustelo, 2005, p. 254). En el nivel de la sobrevivencia, de la materialidad de existir que describe Bustelo, encontramos el

La Revista Estudios es editada por la Universidad de Costa Rica y se distribuye bajo una Licencia Creative Commons Atribución-NoComercial-CompartirIgual 3.0 Costa Rica. Para más información envíe un mensaje a revistaestudios.eeg@ucr.ac.cr. 


\section{Dossier: V Simposio Internacional y IX Nacional sobre Derechos de la Niñez y la Adolescencia}

problema de la infantilización de la pobreza en América Latina. La CEPAL ha descrito cómo este fenómeno comenzó a ser detectado por diversos estudios desde la década de 1990, los cuales evidenciaron la sobre representación de la infancia y la adolescencia en la pobreza, así como la menor reducción de la pobreza infantil con relación a la reducción de la pobreza total de la población (CEPAL, 2013, p. 95).

Para Bustelo, la pobreza infantil mantiene la vida al nivel de la sobrevivencia (zoé), y limita el derecho a participar activamente de la sociedad; es decir, ejercer la vida como bios, como ciudadanía. La biopolítica de la infancia consiste entonces, en "mantenerla en la zoé como solo sobrevivencia e inhibir o regular el desarrollo de la ciudadanía y su acceso a la política" (Bustelo, 2005, p. 256). Parangonando a Giorgio Agamben, Bustelo propone la definición de niño-sacer para describir esta coexistencia entre la infancia sacralizada del paradigma de derechos que se convierte en objeto de protección y la infancia en estado de indefensión, en desamparo social, que puede ser destinataria de diversas formas de violencia impune, como aquellas manifestaciones de incumplimiento de derechos citadas anteriormente.

\section{La infancia generalizada}

Retomando la definición de violencia contra los niños, niñas y adolescentes de la OMS, la cual incluye "acciones u omisiones de individuos, instituciones o colectividades, exista o no intencionalidad de causar un daño o limitar su desarrollo integral" (UNICEF, 2017, p.66), propongo algunas reflexiones sobre las dificultades en el abordaje de los problemas de la infancia dentro del marco del enfoque de derechos.

Una primera dificultad está relacionada con el desconocimiento sobre el estado general de la salud mental de la infancia. El VIII Informe Estado de los Derechos de la Niñez y la Adolescencia en Costa Rica señala la carencia de

\section{(c) (i) (2)}

La Revista Estudios es editada por la Universidad de Costa Rica y se distribuye bajo una Licencia Creative Commons Atribución-NoComercial-CompartirIgual 3.0 Costa Rica. Para más información envíe un mensaje a 
Dossier: V Simposio Internacional y IX Nacional sobre Derechos de la Niñez y la Adolescencia información sobre la salud mental de los niños, niñas y adolescentes de nuestro país:

... el Estado parte no ha proporcionado información ni dato alguno sobre los problemas de salud mental relacionados con los niños, los adolescentes y sus padres, el alcance de dichos problemas y, en su caso, los estudios 0 investigaciones realizados y las medidas preventivas adoptadas, o la accesibilidad de los servicios pertinentes (UNICEF-UCR, 2015, p. 74).

Para quienes trabajan con primera infancia se hace evidente que, en el contexto actual, se enfrentan cada vez mayores dificultades de niñas y niños relacionadas con el lenguaje y la comunicación social. Presenciamos igualmente, una proliferación de diagnósticos sobre trastornos de la infancia ligados a la hiperactividad, desatención y dificultades en la socialización. A nivel mundial, la OMS ha advertido sobre el incremento en las últimas décadas de la prevalencia del Trastorno del Espectro Autista en la infancia (TEA). Se calcula que 1 de cada 160 niños es diagnosticado dentro de esta categoría, aunque debido a falta de estudios en todos los países, esta cifra podría ser aún mayor (Organización Mundial de la Salud, 2018). Si bien la ampliación de los criterios diagnósticos se señala como una de las posibles razones del incremento en la prevalencia del TEA, es interesante contrastar esta ampliación con el desconocimiento y la falta de consenso que aún persiste respecto a las causas de este trastorno.

Las y los profesionales vinculados al campo del desarrollo infantil temprano, enfrentan cada vez más una fuerte demanda de diagnósticos estandarizados y soluciones generalizadas que no necesariamente cumplen la función de orientar los abordajes en el caso por caso, ni responden a la pregunta sobre qué significa el bienestar de la infancia y si esto podría generalizarse para todos los casos. En particular hay dos riesgos en el abordaje de los problemas de la infancia: la patologización de la vida cotidiana y la homogeneización de la infancia.

\section{(c) (i) (2)}

La Revista Estudios es editada por la Universidad de Costa Rica y se distribuye bajo una Licencia Creative Commons Atribución-NoComercial-CompartirIgual 3.0 Costa Rica. Para más información envíe un mensaje a 


\section{Dossier: V Simposio Internacional y IX Nacional sobre Derechos de la Niñez y la Adolescencia}

Desde hace algunos años, varias voces vienen alertando sobre el riesgo de que la proliferación de nuevos diagnósticos pueda producir un proceso de patologización de la infancia (Morell, et al., 2009; Untoiglich, 2014; Ribeiro, 2015). Entre las formas en que este riesgo se expresa están el traslado masivo de los problemas del devenir infantil al campo médico y el enfoque exclusivo sobre las características conductuales observables sin tomar en cuenta la historia particular del infante y sus contextos. En una exposición de los procesos de patologización y medicalización de las infancias en América Latina, Gisela Untoiglich (2014) llama la atención sobre el alto porcentaje de infantes que están siendo medicados cada vez a edades más tempranas. Apunta también sobre el hecho de que se tienda a la confirmación de diagnósticos del desarrollo infantil en función a la respuesta positiva a determinados psicofármacos; situación que evidentemente, plantea importantes problemas clínicos en el tratamiento de estos problemas.

Bianchi (2016) indica que el DSM-V tipificó el Trastorno por Déficit Atencional con Hiperactividad (TDAH) y el TEA identificando factores de riesgo, con el objetivo de enfocarse más específicamente hacia la detección precoz y la prevención. Como consecuencia, se produjo la ampliación de las categorías diagnósticas, acercando la brecha entre normalidad y patología. Si bien estos polos no se llegan a diluir, los diagnósticos tipificados de esta manera se insertan en una lógica de modulación, en la cual las intervenciones apuntan al presente "para asegurar el estado óptimo y el mejor futuro posible de los sujetos" (Bianchi, 2016, p. 427). Para este autor, la ampliación del diagnóstico y su consecuente tratamiento posiciona la infancia como unos de los blancos más importantes de los procesos de biomedicalización de la sociedad. Tomando en cuenta estas consideraciones, podemos pensar que el uso de herramientas para la evaluación de los problemas de la infancia no debería necesariamente excluir la mirada sobre la singularidad y un espacio para la palabra del infante: cómo vive y cómo entiende cada niño y cada niña lo que le sucede.

\section{(c) (i) (2)}

La Revista Estudios es editada por la Universidad de Costa Rica y se distribuye bajo una Licencia Creative Commons Atribución-NoComercial-CompartirIgual 3.0 Costa Rica. Para más información envíe un mensaje a 
Dossier: V Simposio Internacional y IX Nacional sobre Derechos de la Niñez y la Adolescencia

Por ejemplo, el cuestionario M-CHAT (Cuestionario de Autismo en la Infancia- Modificado) para la detección del espectro autista, está diseñado para que los padres o las madres lo completen sin necesidad siquiera de que el profesional tenga contacto con el infante y es con base en el puntaje obtenido de esta manera que se plantea el riesgo de autismo. Si bien el uso difundido de esta herramienta ha representado un gran aporte a la necesaria detección precoz de problemas del desarrollo, es importante reflexionar sobre el uso mecánico de instrumentos diagnósticos, que dejan por fuera otros aspectos que podrían facilitar una visión más integradora en la valoración clínica. Esto a fin de evitar un etiquetamiento temprano de las manifestaciones infantiles, especialmente ante el riesgo de medicalizar procesos de la infancia que podrían ser respuestas o intentos de solución singulares de un niño o una niña ante su condición y contexto de vida.

Es importante considerar que la realidad de muchas dificultades de la infancia no es exclusivamente biológica, también es discursiva; es decir, que los problemas hablan, quieren decir algo sobre el sufrimiento o la imposibilidad de un niño o una niña ante su realidad. Las razones por las cuales un niño no aprende, no logra concentrarse, no se queda quieto o no se comporta según lo esperado, pueden ser muy diversas e intrincadas. Esto nos convoca a la responsabilidad de estar alertas ante la simplificación diagnóstica de problemas complejos.

De aquí se desprende el segundo riesgo: la homogenización de las dificultades de la infancia. Cuando el saber sobre la infancia se usa para realizar intervenciones desde una perspectiva adultocéntrica y totalizadora, sin abrir espacios para la palabra y la singularidad del niño o la niña, nos acercamos a lo que Ana Ruth Najles (2010) Ilama la evaluación generalizada. Con este concepto, la autora describe la tendencia a homologar científicamente los padecimientos infantiles y hacerlos equivalentes, "cuando se trata de evaluar a los seres hablantes en masa y en detalle, en cuerpo y alma, estamos ante una operación de control,

\section{(c) (i) (9)}

La Revista Estudios es editada por la Universidad de Costa Rica y se distribuye bajo una Licencia Creative Commons Atribución-NoComercial-CompartirIgual 3.0 Costa Rica. Para más información envíe un mensaje a revistaestudios.eeg@ucr.ac.cr. 
Dossier: V Simposio Internacional y IX Nacional sobre Derechos de la Niñez y la Adolescencia que ataca el derecho al secreto, que es lo único que puede oponer resistencia al control" (Sección de conclusiones, párr.9). Para esta autora, a través de la evaluación generalizada la particularidad del sujeto a ser evaluado queda oculta bajo la medición, de modo que un ser único pasa a ser uno más entre otros homologables.

\section{Al rescate de la singularidad}

La infancia generalizada es también una infancia silenciada, en la medida en que la evaluación y el tratamiento de sus dificultades se sustentan exclusivamente en descripciones conductuales, sin detenerse en la escucha, en la indagación de contextos y las historias particulares, para intentar comprender de un modo más amplio lo que le sucede a cada niño o niña.

Aunque es innegable la importancia de los avances en la caracterización temprana de las dificultades de la infancia que han permitido afinar los criterios diagnósticos, la cautela frente al riesgo de homogeneización convoca a evitar el intento de atrapar dentro de categorías únicas lo que no es homogenizable. La particularidad de cada niño y cada niña no se puede reducir a la medida o a la comparación, hay una parte de no saber que se resta al conocimiento que tienen los adultos sobre la infancia y que no se resuelve con la ampliación de diagnósticos. Por el contrario, es necesario defender y sostener ese espacio de desconocimiento, para que lo pueda complementar cada infante con su particularidad, tolerar una parte no calculable en nuestro saber sobre la infancia para poder trabajar con ella.

Desde la perspectiva de derechos, las personas adultas deben evitar toda voluntad de dominio sobre la infancia y esto incluye el intento de operar desde un saber totalizador. Ante los riesgos de patologización y homogeneización de la infancia, es necesario tomar distancia de las generalizaciones clasificatorias para

\section{(c) (i) (2)}

La Revista Estudios es editada por la Universidad de Costa Rica y se distribuye bajo una Licencia Creative Commons Atribución-NoComercial-CompartirIgual 3.0 Costa Rica. Para más información envíe un mensaje a 
Dossier: V Simposio Internacional y IX Nacional sobre Derechos de la Niñez y la Adolescencia

comprender su sufrimiento y dificultades de una manera que permita incluir lo particular de cada niño y cada niña.

En síntesis, no se trata de intervenir sin conocimientos sobre la infancia, tampoco de un intento imposible por permanecer fuera del empuje de nuestros tiempos, ya que no podemos pensarnos como no afectados por las coordenadas de la época que se filtran en el quehacer cotidiano, ni por fuera del riesgo de reproducirlas en prácticas habituales. Pero es posible intentar no ser ingenuos respecto a los saberes que nos atraviesan y que sostenemos como profesionales en el campo de la infancia, poder colocarnos un poco al margen para mantener alguna interrogación permanente sobre nuestra práctica e intentar construir con cada niño y cada niña, subjetividad y existencia.

\section{Referencias bibliográficas}

Bianchi, E. (2016). Diagnósticos psiquiátricos infantiles, biomedicalización y DSM: ¿hacia una nueva (a)normalidad? Revista Latinoamericana de Ciencias Sociales, Niñez y Juventud, 14(1), 417-430. Recuperado de: https://dx.doi.org/10.11600/1692715x.14128210715

Bustelo, E. (2007). El recreo de la infancia. Argumentos para otros comienzos. Buenos Aires: Siglo XXI.

. (2005). Infancia en Indefensión. Salud colectiva [online]. 1(3), 253-

284. Recuperado de: https://dialnet.unirioja.es > descarga > articulo > 2511292

CEPAL (2013). Panorama Social de América Latina. Santiago de Chile: Naciones Unidas

\section{(c) (i) (2) (2)}

La Revista Estudios es editada por la Universidad de Costa Rica y se distribuye bajo una Licencia Creative Commons Atribución-NoComercial-CompartirIgual 3.0 Costa Rica. Para más información envíe un mensaje a revistaestudios.eeg@ucr.ac.cr. 
Dossier: V Simposio Internacional y IX Nacional sobre Derechos de la Niñez y la Adolescencia

Han, B. (2012). La sociedad del cansancio. Argentina: Herder

Matarrita Chaves, F. (11 de agosto de 2019). Las crueles historias de maltrato infantil: Los niños y niñas que sufren en Costa Rica. La Nación en línea, Revista Dominical.

Morell, M.E., Martínez, C. y Quintana, J.L. (2009). Disease mongering, el lucrativo negocio de la promoción de enfermedades. Pediatría Atención Primaria, 11(43), 491-512. Recuperado de: http://scielo.isciii.es/scielo.php?script=sci arttext\&pid=S1139$\underline{76322009000400011 \& \operatorname{lng}=e s \& t \operatorname{lng}=e s .}$

Najles, A. (2010). El niño del mercado global. Conferencia llevada a cabo en la Facultad de Filosofía y Letras de la UNAM. Nueva Escuela Lacaniana del Campo Freudiano, Ciudad de México. Recuperado de: http://www.nelmexico.org/index.php?sec=Actividades-internacionales\&file=Actividadesinternacionales/Textos-Conferencias/10-06-04 El-nino-del-mercadoglobal.html

OMS (2018). Nota de prensa: Trastornos del espectro autista. 02 de abril de 2018. Recuperado de: https://www.who.int/es/news-room/fact-sheets/detail/autismspectrum-disorders

Organización Mundial de la Salud/Organización Panamericana de la Salud (2017). Nota de prensa: "Depresión: hablemos", dice la OMS, mientras la depresión encabeza la lista de causas de enfermedad. Ginebra / Washington, 30 de marzo de 2017.2 Recuperado de: https://www.paho.org/hq/index.php?option=com content\&view=article\&id=13 272:qdepresion-hablemosq-dice-la-oms-mientras-la-depresion-encabeza-lalista-de-causas-de-enfermedad\&ltemid=42050\&lang=es

\section{(C) $(0 \bigcirc$}

La Revista Estudios es editada por la Universidad de Costa Rica y se distribuye bajo una Licencia Creative Commons Atribución-NoComercial-CompartirIgual 3.0 Costa Rica. Para más información envíe un mensaje a revistaestudios.eeg@ucr.ac.cr. 
Dossier: V Simposio Internacional y IX Nacional sobre Derechos de la Niñez y la Adolescencia

Ribeiro, R. (2015). Patologización de la infancia cotidiana. Teoría y Crítica de la Psicología, 5, 148-156. Recuperado de: http://www.teocripsi.com/ojs/index.php/TCP/article/view/17

Soler, C. (2010). Los estados depresivos. AUN Revista del Foro Analítico del Río de la Plata, 2(3 y 4), 13-32.

UNICEF (2017). Estado Mundial de la Infancia 2017. Niños en un mundo digital. Fondo de las Naciones Unidas para la Infancia, diciembre de 2017. Disponible en: https://www.unicef.org/spanish/sowc2017/

UNICEF-UCR, (2015). VIII Informe Estado de los derechos de la Niñez y la Adolescencia en Costa Rica.

Untoiglich, G. (2014). Medicalización y patologización de la vida: situación de las infancias en Latinoamérica. Nuances: estudos sobre Educacao, 25(1), p.2038. Recuperado de: http://revista.fct.unesp.br/index.php/Nuances/article/view/2743

Vargas, M. (2012). Muertes por agresión infantil en Costa Rica entre los años 2003 y 2011. Medicina Legal de Costa Rica, 29(2), 07-18. Recuperado de: https://www.scielo.sa.cr/scielo.php?script=sci arttext\&pid=S1409$\underline{00152012000200002}$ 